\title{
Mujer y piragua: estudio de las variables moduladoras del abandono deportivo de las mujeres piragüistas en modalidades olímpicas \\ Women and canoeing: study of the variables modulating withdrawal in canoeists women in Olympic modalities \\ *Manuel Isoma Folgar, **MartaFelpeto Lamas, *DiegoAlonso Fernández, ***Patricia Gómez Salgado, ***AntonioRialBoubeta \\ *Universidad de Vigo (España), **Real Federación Española de Piragüismo (España), ***Universidad Santiago de Compostela (España)
}

Resumen: La práctica físico-deportiva es un elemento valioso en la salud integral de los individuos. Sin embargo, el abandono deportivo es un fenómeno que prolifera y se acentúa en las etapas de formación sobre todo en las practicantes femeninas. Los motivos del abandono son múltiples y diversos, estando muy marcados por las características de la modalidad deportiva y de sus practicantes. El piragüismo en una modalidad muy exigente y competitiva que en el último lustro en nuestro país ha mostrado una tasa de abandono muy elevada (del 32,9\% al 49,7\%) entre las deportistas femeninas federadas. El presente estudio pretende reconocer los motivos por los cuales las piragüistas han abandonado la práctica contribuyendo a establecer medidas y recomendaciones que atenúen esta realidad en el futuro. Para ello se ha recurrido a una estrategia mixta, combinando un enfoque cualitativo y cuantitativo. El estudio cualitativo se basó en dos Grupos Focales, uno con ex-palistas (n=6) y otro con entrenadoras/es (n=8), mientras que el estudio cuantitativo consistió en un cuestionario ad hoc realizado telefónicamente entre deportistas que abandonaron la modalidad en los tres último años $(\mathrm{n}=160)$. Los resultados obtenidos permiten afirmar que el abandono femenino tiene un carácter multifactorial pero está muy determinado por los roles de género vigentes que infravaloran a la mujer palista, la excesiva presión competitiva, la dificultad para conciliar el piragüismo y los estudios, así como la carencia de categorías de menor nivel competitivo. Las Federaciones y Administraciones deberían realizar un mayor esfuerzo en labores de promoción del piragüismo femenino.

Palabras clave: piragüismo, mujer, abandono deportivo, deporte federado.

Abstract: The physical-sports practice is valuable for any individual's overall health. However, dropout from sports is an increasing phenomenon accentuated in the formative stages, especially in female practitioners. Reasons for withdrawing are diverse and highly dependent on the characteristics of each sport and its practitioners. Canoeing in a very demanding and competitive discipline that has shown a very high dropout rate (32.9\% to $49.7 \%$ ) among federated female athletes from our country in the last five years. This study aims to recognize the reasons why canoeists abandon the practice so to help establishing measures and recommendations to mitigate this occurrence in the future. A mixed strategy, combining qualitative and quantitative approach, was employed for the study purposes. The qualitative study was based on two focus groups, one composed by ex-paddlers (n = 6) and one with coaches/s ( $\mathrm{n}=8$ ), while the quantitative study consisted of an ad-hoc questionnaire conducted by telephone with athletes who withdrew from that sport within the last three years $(\mathrm{n}=160)$. The results confirm that women's dropout has a multifactorial nature, yet it is largely determined by gender biases commonly underestimating paddler women, excessive competitive pressure, difficulty in pairing canoeing and education, and lack of categories with lower competitive level. Federations and authorities should make greater efforts in promoting women's canoeing modality.

Keywords: canoeing, women, sport dropout, federated sport.

\section{Introducción}

En la literatura científica es un hecho constado que la práctica regular de actividad físico-deportiva constituye una variable positiva en la salud física (Jiménez, Martínez, Miró \& Sánchez, 2008; Moreno, Cervelló \& Moreno, 2008), psíquica y social (Edmuns, Ntoumanis \& Duda, 2007) de los individuos. Sin embargo, a pesar de estos potenciales beneficios algunos trabajos recientes han observado como cada vez más jóvenes abandonan la práctica físico-deportiva regular (Gómez, Ruiz \& Pieron, 2010; Isorma, Ruiz \& Rial, 2013).

Existen diferentes modelos o teorías que intentan explicar la adhesión y el abandono de la práctica físico-deportiva. Algunos ponen énfasis en el aspecto psicológico o individual del sujeto, mientras otros se centran más en el componente social donde este se desenvuelve(Chillón, 2005). No obstante, existe una variable que influye altamente tanto en la práctica como en el abandono: el género del practicante.

Diversos trabajos han observado diferencias significativas en la práctica físico-deportiva en función del género, siendo los hombres los que obtienen valores más elevados de práctica (García-Ferrando, 2005; Isorna, Rial \& Vaquero-Cristóbal, 2014; Baena \& Rebollo, 2008; Pavón \& Moreno, 2008; Macarro, Romero y Torres, 2010; Ruiz, Isorna, Ruiz-Risueño \& Vaquero, 2014).Anivel europeo, esta tendencia también semanifiesta, con una proporción de mujeres inactivas significativamente más alta que la de los hombres (Mota \& Silva, 1999; Piéron, Telama, Almond \& Carreiro da Costa, 1999; Sallis, Prochaska \& Taylor, 2000). En nuestro contexto próximo, España presenta una realidad similar con una población femenina más sedentaria que la masculina (GarcíaFerrando, 2006; Martínez et al., 2012; Mollá, 2007; Moreno, Pardo, Jorge, \& Huéscar, 2016).

La incorporación de la mujer a ciertas modalidades y ámbitos deportivos ha sido progresiva y en ocasiones muy lenta debido a las adaptaciones de su rol y entorno social (Puig, 2001). La literatura recoge como barreras (internas y externas), principalmente, la tradición cultural de muchas modalidades deportivas, siendo muchas de ellas eminentemente masculinas (en tiempos, espacios, lenguaje o símbolos); los conflictos de rol o de identidad de género asociados; las diferencias en retribución económica según el género del o la deportista; la falta de modelos femeninos en los medios de comunicación o la escasa representación de las mujeres en los órganos de decisión (Lavallee \& Wylleman, 2000; Pallarès, Azócar, Korte, Ramis, Fuentes \& Torregrosa, 2009). En efecto, la evidencia cotidiana de los medios de comunicación, nos muestran que es considerable el esfuerzo que realizan muchas de las deportistas para compatibilizar su carrera en la competición con la profesional; y las dificultades que estas experimentan en los ámbitos deportivos en su retirada y cara a la posterior adaptación a una carrera profesional alternativa en el ámbito laboral deportivo (Selva, Pallarès \& González, 2013).

Paralelamente, el abandono de la práctica físico-deportiva discurre por un sendero muy similar siendo casi el doble entre las mujeres que entre los hombres (Isorna, Ruíz \& Rial, 2013; Lee, Fredenburg, Belcher \& Cleveland, 1999; Pavón, Moreno, Gutiérrez \& Sicila, 2004). Esta circunstancia también sucede en la práctica deportiva federada, unámbito en donde la mujer presenta históricamente una cuota de participación menor que el hombre (García Ferrando, 2006). En contextos concretos, «a priori», más igualitarios como el universitario, también se aprecia un mayor número de hombres federados que de mujeres (Castañeda, Zagalaz, Chacón, Cachón \& Romero, 2014). 
La práctica deportiva competitiva exige un nivel de compromiso más elevado para el deportista, por lo que la incidencia del abandono es igualmente preocupante y más en el caso de las mujeres. Los motivos de abandono son múltiples y muy variados (Isorna, Ruíz \& Rial, 2013). El motivo más aludido sobre todo por los adolescentes/jóvenes suele ser la falta de tiempo seguido muy de cerca por la preferencia en la realización de otras actividades de tiempo libre, así como la pereza o desgana (Macarro, Romero \& Torres, 2010). Otras explicaciones ponen el énfasis en la influencia de los estereotipos de género en la sociedad y la enseñanza, que terminan por generar diferencias tanto de intereses como de motivaciones, así como en el grado de práctica de ciertas modalidades deportivas (Binachi \& Brinnitzer, 2000; Moreno, Martínez \& Alonso, 2006; Scraton \& Flintoff, 2002). En esta línea, en un estudio elaborado con mujeres andaluzas que habían abandonado la práctica deportiva de Alarcón (2008), observamos que el mayor porcentaje estaba vinculado a las responsabilidades relacionadas con el hogar y los hijos (73\%), un motivo muy ligado al rol de «cuidadora» que la mujer aún tiene en nuestra sociedad. Etapas tan cruciales como la formación universitaria y su difícil acomodación a las rutinas de un deportista de alto nivel acaban siendo un motivo de abandono importante (Álvarez, Pérez, González \& López, 2014; Latorre, García, \& López, 2017).

Otros motivos para el abandono del deporte son el carácter altamente competitivo de las pruebas federadas de categorías inferiores (Orlick \& Botterill, 1975), con el consiguiente énfasis en la «victoria a cualquier precio», la deficiente estructura administrativa y de recursos disponibles, las mesetas en el rendimiento y/o en el aprendizaje y el conflicto de intereses, este último como el más frecuente (Rodríguez, 2000). Otros autores como Ogilvie \& Howe (1991) señalan tres factores principales para el cese o retirada de una actividad deportiva: el proceso de selección, el cual se da a todos los niveles de competición, la edad cronológica y la lesión deportiva.

En otras ocasiones se han determinado como principales motivos para el cese de la actividad deportiva la excesiva presión de la competición y el rendimiento (Ponseti, García, Cantallops \& Vidal, 2016), la falta de interés y consideración que el deporte no era divertido, otras aficiones no deportivas y los problemas con el entrenador/a (Saura, 1996). En estesentido, debemos resaltar la importancia dela actuación del entrenador/ a que puede tornarse como un agente esencial tanto para favorecer la continuidad de la práctica, como para conducir al abandono de la misma (Sousa, Cruz, Viladrich, \& Torregrosa, 2007). Sería un error obviar que independientemente de la motivación que las deportistas perciben a través del programa de contenidos propuesto por el entrenador/a, las relaciones que se establecen entre este y las deportistas son decisivas y determinantes (Torre, Cárdenas y García, 2001; Torregrosa, Sousa, Viladrich, Villamarín \& Cruz, 2008). Por este motivo, resulta especialmente relevante el asesoramiento psicológico a entrenadores (Cruz, 1997) y se debe resaltar la importancia que puede llegar a adquirir el comportamiento del técnico en su relación con la deportista (Tuero \& Salguero 2001a; 2001b).

Todo ello cobra especial trascendencia en un deporte como el piragüismo, caracterizado desde hace décadas por elevadas tasas de abandono y enormes dificultades desde el punto de vista de los responsables federativos para garantizar niveles de adherencia similares a los de otros deportes, especialmente en el paso de la adolescencia a la adultez. En el caso del piragüismo femenino, la realidad es si cabe aún más preocupante, con tasas de abandono que llegan a superar en algunas temporadas el $40 \%$ tal y como se puede observar a continuación en los datos aportados por la Real Federación Española de Piragüismo.

La investigación científica tanto en el campo de las Ciencias de la Actividad Física y el Deporte como en la ciencia en general ha tenido tradicionalmente una visión androcéntrica del conocimiento. Tal y como recoge Vilariño \& Villar (2012), esta postura sesgada ha supuesto la exclusión y la «invisibilización» de las mujeres, contribuyendo a un conocimiento más limitado de su realidad y necesidades.

Un error que se detecta al analizar los diferentes estudios es que han sido creados con preguntas cerradas que no incluyen posibilidades de

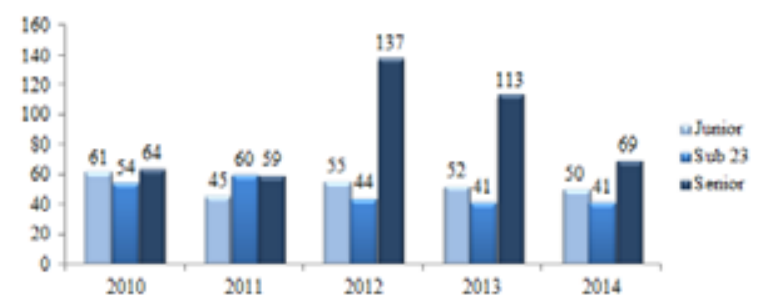

Figurn 1.N" de mojeres que abandonaron el piragliaeo foderado entre 2010 y 2014 (Fvente: Real Fodencioie Espalota de Pingliseo)

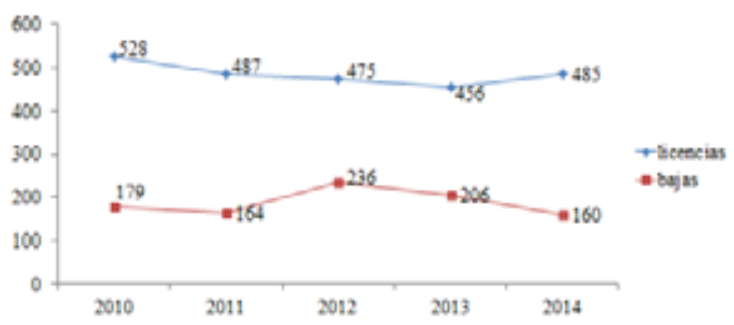

Figurn 2. No de licencins fodentivas de mujeres y No de brias entre 2010 y 2014

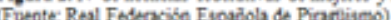

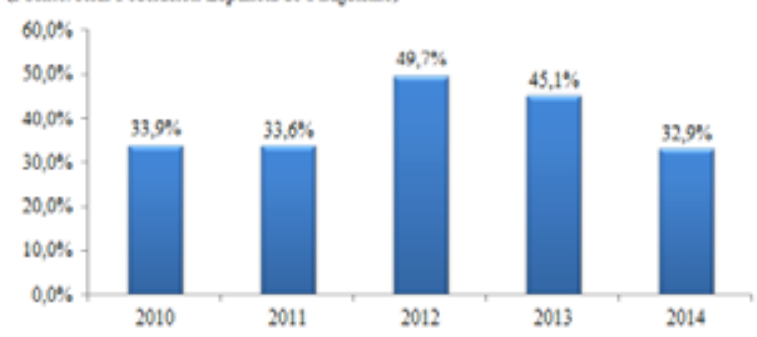

Figura 3. Taus de tiundono de muperes entre 2010 y 2014 (Fopente Real Federación Espariola de Piraglismo)

acomodación desde una perspectiva de género, introduciendo así un sesgo. Por tanto, es inevitable pensar que preguntando lo mismo a hombres y a mujeres, nunca daremos con la clave de una problemática como el abandono que claramente está influenciada por las connotaciones sociales vinculadas al género de los individuos.

Conocer de primera mano el sentir de las propias deportistas, en este caso piragüistas y hacer aflorar las verdaderas razones de su abandono, se hace más quenunca una tarea obligada para intentar revertir la alta tasa de abandono femenino en esta modalidad deportiva que, por otro lado, ha dado tantas alegrías al deporte nacional en estos último años, con numerosas victorias a nivel internacional.

\section{Objetivos}

El presente estudio pretende reconocer los motivos por los cuales las piragüistas han abandonado la práctica de esta modalidad deportiva, explorando las opciones y percepciones tanto de las propias deportistas, como de sus entrenadores/as. La información recabada pretende contribuir a establecer medidas y recomendaciones para que las federaciones puedan reducir las elevadas tasas de abandono presentes en el piragüismo femenino español

\section{Metodología}

Se ha optado por recurrir a una estrategia mixta, combinando un enfoque cualitativo y un enfoque cuantitativo.

A nivel cualitativo se realizaron dos Grupos Focales (Kitzinger, 1995; Morgan 1997), uno con ex-palistas y otro con entrenadores/as. A nivel cuantitativo, se realizó una encuesta entre deportistas de categoría junior, sub-23 y senior que abandonaron la práctica activa del piragüismo a nivel federado en los tres últimos años. Para optimizar el aprovechamiento de toda la información se realizaron antes los grupos focales y posteriormente la encuesta. 


\section{Estudio Cualitativo}

\section{Participantes}

En el grupo de ex-palistas participaron 6 mujeres, todas ellas de la comunidad gallega y con edades comprendidas entre 22 y los 30 años. El grupo de entrenadoras/es estuvo compuesto por 8 personas (6 mujeres y 2 hombres), con edades comprendidas entre 28 y 57 años, pertenecientes también a diferentes clubes de la comunidad gallega.

\section{Materiales}

Las dinámicas grupales de los grupos focales fueron grabadas en audio, con la debida aprobación de los asistentes, garantizando la confidencialidad de la información recogida. Se utilizaron como apoyo al debate diferentes materiales (cartulinas, fichas...) para mejorar la implicación, el dinamismo y el equilibrio de la participación.

\section{Procedimiento}

Las sesiones de grupo tuvieron una duración aproximada de dos horas, estructurándose en 4 grandes bloques:

1. Historia personal de Práctica Deportiva

2. Práctica de Piragüismo (motivos de elección, valores diferenciales, dificultades, etc.)

3. Motivos de Abandono

4. Claves para la promoción del piragüismo femenino y apoyo a las deportistas

\section{Triangulación y análisis de datos}

Las sesiones de grupo fueron moderadas por dos investigadores, quienes analizaron individualmente las transcripciones y categorizaron lainformación de forma independiente, dotando así de validez concurrente a los temas y subtemas establecidos (Savin-Baden \& Howell, 2013).

\section{Estudio Cuantitativo}

\section{Participantes}

El universo de referencia comprendía a toda la población de mujeres que abandonaron la práctica del piragüismo con licencia federativa en las tres últimas temporadas (desde 2012, 2013 y 2014). Según datos de la propia Federación Española de Piragüismo la población objeto de estudio asciende a 497 palistas (219 que abandonaron siendo senior, 126 siendo sub-23 y 152 tras competir en categoría junior). Se intentó establecer contacto con la totalidad de la población objeto de estudio (487 expalistas cuyos datos aparecen recogidos en la base de datos de la Federación Española de Piragüismo). No obstante, el contacto resultó efectivo en tan sólo 160 casos, que compusieron la muestra final para el análisis. Su edades estaban comprendidas entre los 17 y los 40 años ( $\bar{X}=23.46 ; S_{X}=4.66$ ). El 50,6\% abandonaron la práctica del piragüismo en categoría senior, el 13,9\% siendo sub-23 y el 35,4\% restante siendo todavía deportistas junior. La ficha técnica del estudio se recoge en la siguiente tabla.

Tabla 1.

Ficha técnica del estudio cuantitativo

Ámbito: España

Universo: Finito. 497 mujeres que abandonaron la práctica del piragüismo con licencia

federativa en las tres últimas temporadas

Tipo de muestreo: Aleatorio, estratificado con afijación proporcional según Categorí

Tamaño de la muestra: 160 entrevistas

Instrumento de recogida de datos: Cuestionario "ad hoc"

Procedimiento: Entrevista telefónica

\section{Procedimiento}

Los datos fueron recogidos mediante una entrevista telefónica estructurada, por un equipo de cuatro entrevistadoras con dilatada experiencia en este tipo de estudios. Las entrevistas fueron realizadas con la ayuda de un cuestionario elaborado específicamente que incluía una breve introducción orientada a conseguir la mayor implicación y sinceridad posible en las respuestas. Se incluyeron también ítems de tipo sociodemográfico y se reservó un espacio para recoger las observaciones o incidencias de la entrevistadora sobre el proceso. La duración de las entrevistas osciló entre 20 y 25 minutos y fueron realizadas durante el mes de noviembre de 2015.

\section{Resultados}

\section{Estudio cualitativo}

Tras analizar los discursos tanto de palistas como de entrenadoras, es posible comprobar que los motivos a los que se atribuye el abandono del piragüismo son múltiples y muy variados (Tabla 2). Estamos, por tanto, ante un problema complejo y multidimensional, que cada deportista vivencia de una manera particular.

Tabla 2.

Listado de motivos de abandono

\begin{tabular}{|l|}
\hline \multicolumn{1}{|c|}{ MOTIVOS DE ABANDONO } \\
\hline \multicolumn{1}{|c|}{ PALISTAS } \\
\hline -Discriminación con respecto a los hombres. (Falta de oportunidades para ser internacional) \\
-Falta o pérdida del apoyo de los padres \\
-Estudios fuera del lugar de residencia \\
-Exceso de competiciones y del nivel las competiciones \\
-Dificultades para entrenar por falta de tiempo \\
-Demasiado sacrificio para poco beneficio \\
-Lesiones \\
-Falta de tiempo o conflicto de intereses con otras actividades \\
-Miedo al fracaso, a no alcanzar los resultados anteriores \\
-Estereotipos del cuerpo femenino ligado a las mujeres piragüistas. \\
-Elevado nivel de las competiciones \\
-Cansancio físico después del entrenamiento que dificulta atender a otras obligaciones \\
-Atender a nuevas relaciones sociales (novio y amigas) \\
\hline \\
\hline -Estereotipos del cuerpo de las piragüistas \\
-Influencia de los padres \\
-Presión competitiva, siempre hay que competir al máximo nivel \\
-Excesivo requerimiento de tiempo de los entrenamientos que les impide realizar otras \\
actividades \\
-Coste del material (especialmente en slalom) \\
-La comparación con los otros y no con uno mismo (siempre se comparan los resultados con \\
los de los demás no con la propia evolución). \\
-Lesiones físicas y cansancio psicológico \\
-No existe diferenciación de niveles (no hay final B y C) \\
-Estudios, incorporación a la universidad \\
-Excesivo sacrificio y poca recompensa \\
-Problemas con el entrenador/a y los directivos/as \\
-Problemas de convivencia entre compañeras \\
-Falta de apoyo familiar \\
-Falta de tiempo que les impide realizar otras actividades \\
-Tener pareja \\
-Miedo al fracaso, bajada de nivel \\
\hline
\end{tabular}

En la siguiente figura se resumen las diferentes propuestas que tanto palistas como entrenadoras/es realizan para intentar atenuar las tasas de abandono existentes. Como se puede observar, existe una amplia coincidencia entre ex-palistas y entrenadoras/es a la hora de señalar tanto factores sociales, como logísticos y organizativos, que tienen mucho que ver también con el propio funcionamiento de los clubes.

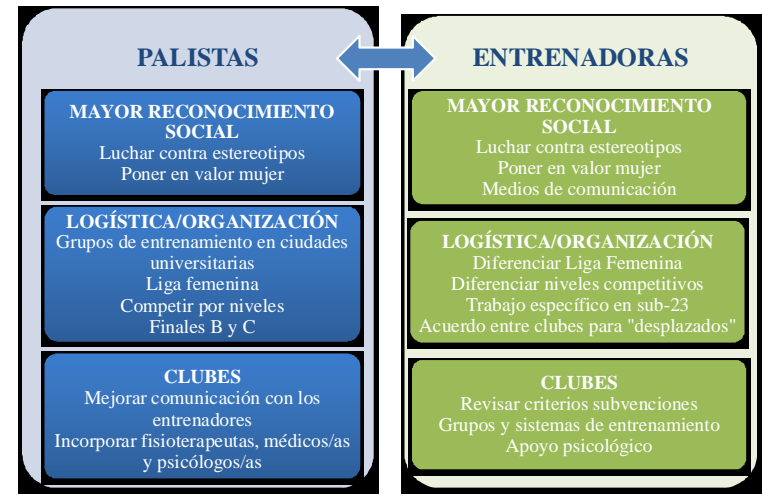

Figura 4.

Medidas para luchar contra el abandono

\section{Estudio cuantitativo}

\section{Piragüismoy Género}

El 31\% de las palistas entrevistadas cree que a día de hoy en España resulta más complejo ser piragüista siendo mujer, mientras que el 64,6\% opina que es difícil para ambos géneros por igual, y solo un 4,4\% 
considera que son los hombres quienes lo tienen más difícil. Aquellas quemantienen que las mujeres lotienen más difícil destacan los siguientes argumentos.

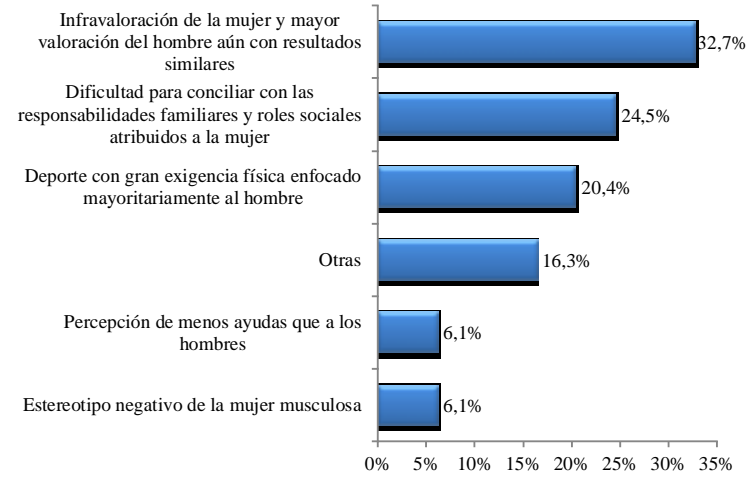

Figura 5. ¿Por qué cree que a día de hoy es más difícil ser piragüista para una mujer?

\section{Motivos de abandono}

A continuación, se analizaron las causas del abandono del piragüismo federado.

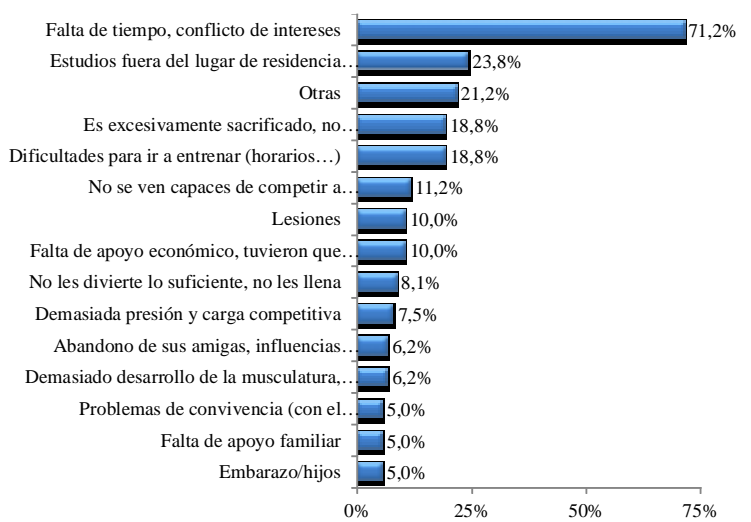

Figura 6. ¿Por qué abandonó el piragüismo federado?

Pese a todo, al 94,4\% de las mujeres les hubiera gustado continuar con su práctica. Este dato no presenta diferencias significativas en función de la categoría deportiva $\left(\div^{2}=1.825 ; p=.401\right)$, tal y como muestra la siguiente figura.

\section{Valoración de la situación actual}

En este epígrafe, se realiza una breve valoración por parte de las mujeres de la situación actual del piragüismo en España, observándose una opinión clara que refleja una baja percepción de la promoción del piragüismo en el territorio nacional, sobretodo, el de categoría femenina.

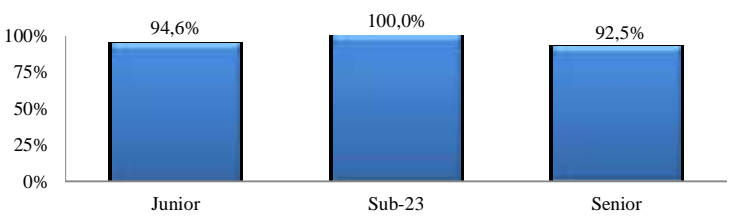

Figura 7. Ex-palistas a las que les hubiera gustado seguir en el piragüismo federado

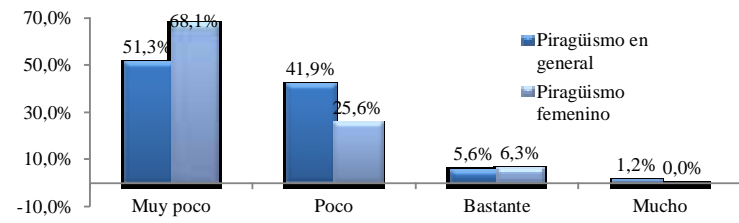

Figura 8. ¿Cuánto cree que se está promocionando el piragüismo en nuestro país?, ¿y el piragüismo femenino en particular?

\section{Recomendaciones y medidas}

Por último, se apuntan cuáles son los retos pendientes en este ámbito bajo el punto de vista de las propias mujeres (Figura 9), y cuáles son las medidas que, en su opinión, habrían de ser susceptibles de tener en cuenta para resolver el problema del abandono femenino (Figura 10).

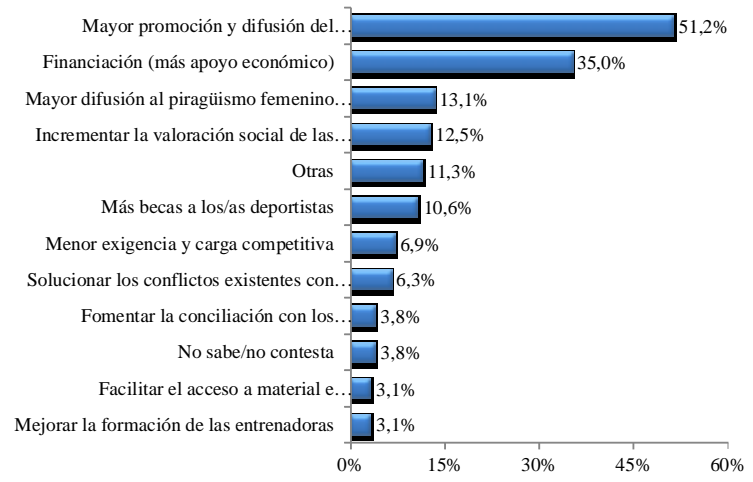

Figura 9. ¿Cuáles son a su juicio las grandes asignaturas pendientes?, ¿qué sugeriría?

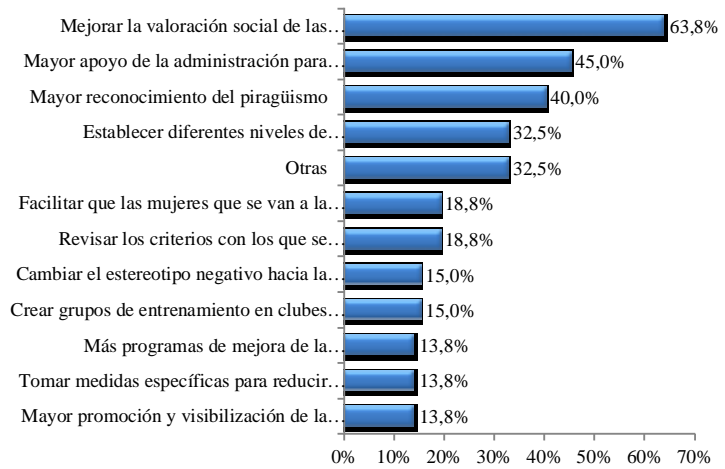

Figura 10. ¿Qué medidas habría que tomar para resolver el problema del abandono femenino en particular?

\section{Discusión}

Los resultados obtenidos en el presente estudio corroboran tendencias sobre el abandono deportivo observadas en la literatura científica anterior pero, la utilización novedosa de dos estrategias empíricas complementarias nos han permitido extraer conclusiones concretas y valiosas sobre los motivos del abandono del piragüismo femenino federado.

Los motivos de abandono señalados por las piragüistas y las entrenadoras son múltiples y variados, en la línea de estudios anteriores (Isorna, Ruíz \& Rial, 2013; Álvarez, Pérez, González \& López, 2014), acentuando asíla necesidad de realizar estudios en ámbitos y modalidades concretas que ayuden a generar un conocimiento real y aplicable.

Los estereotipos de género y el rol femenino actual parecen tener un peso específico en los motivos de abandono de las deportistas algo de lo que también se dejaba constancia en estudios como los de Alarcón (2008) y Moreno, Martínez \& Alonso (2006). Si además de las responsabilidades sociales/familiares que implica el rol femenino se le suman las responsabilidades académicas, este período también tiene una gran trascendencia en el abandono deportivo de las piragüistas, con resultados similares a los obtenidos por Álvarez, Pérez, González \& López (2014) y Álvarez-Pérez \& López-Aguilar (2017). Estas variables confluyen en un «conflicto de intereses» para la deportista que le «empuja» hacia el abandono tal y como ya señalaba Rodríguez (2000) en su estudio como deportistas adolescentes. Es importante destacar que la mayor parte de las deportistas de alto nivel, en comparación con los hombres, han seguido estudios de nivel superior, ya sea módulos formativos-profesionales o universitarios, este hecho implica mucho esfuerzo y mayor sacrificio por parte de ellas, lo cual genera una amenaza 
constante y un obstáculos para continuar la carrera deportiva (González \&Torregrosa, 2009; Selva, Sahagún \& Pallarès, 2011; Torregrosa, Sánchez \& Cruz, 2004 y Wurth, Lee \& Alfermann, 2004).

Sin duda otra circunstancia que parece determinante en el abandono del piragüismo femenino federado es su carácter altamente competitivo y la presión que esto genera en las deportistas. Este motivo ya ha sido señalado anteriormente en la literatura (Ogilvie \& Howe, 1991; Orkick \& Botterill, 1975; Saura, 1996) y parece ligado aún más, si cabe, a una modalidad como el piragüismo en donde la responsabilidad individual en el rendimiento es máxima y no se diluye o comparte con un colectivo, como sucede en los deportes de equipo.

De acuerdo, también, con lo apuntado por Pallarès et al., (2008), entre los motivos del abandono de forma explícita se añaden el menor reconocimiento social de la piragüista, la menor visibilidad y la obtención de menos recursos por ser deporte femenino. Estas desigualdades se convierten en claros obstáculos para continuar con la práctica deportiva generando, a su vez, tal y como indicábamos anteriormente, en grandes dificultades para conciliar el espacio deportivo, el académico y el laboral. No debemos olvidar que las mujeres, en este sentido, planifican en mayor medida su retirada que los hombres (Pallarès, Azócar, Torregrosa, Selva \& Ramis, 2011).

En menor medida, el caso de la maternidad es una de las situaciones donde ellas describen como un ejemplo de clara desigualdad: abandono por parte de las entidades deportivas cuando se tiene un hijo o cuando no se tienen los resultados deportivos esperados post maternidad. Aunque hay un esfuerzo por parte de diferentes organismos por ofrecer una ayuda durante los períodos de maternidad, no todas las mujeres deportistas pueden contar con ellas (Selva et al., 2013).

De este modo y, tras el análisis de los resultados obtenidos, el presente estudio puede aportar las siguientes conclusiones y recomendaciones con el objetivo de contribuir a la minoración del abandono deportivo existente en el piragüismo femenino federado de nuestro país:

- El problema del abandono tiene un carácter multidimensional, es complejo y está sujeto a una enorme variabilidad personal. Se hace necesario el estudio de modalidades y colectivos concretos para obtener conclusiones válidas que puedan traducirse en acciones reales y efectivas.

- Practicar el piragüismo de forma activa y federada no debiera ser sinónimo de competición de alto nivel. A más del 94\% de las expalistas les hubiese gustado seguir federadas pero con una exigencia competitiva y de dedicación menor.

- Las lesiones y la gran exigencia física y psicológica a la que se ven sometidas las deportistas hacen realmente difícil no abandonar. Federaciones y clubes deben trabajar en incorporar figuras profesionales como el/la fisioterapeuta, el médico/a y el psicólogo/a en el apoyo y seguimiento de los/as piragüistas.

- La infravaloración de la mujer palista frente al hombre palista y la difícil conciliación con las responsabilidades familiares y los roles sociales asociados a las mujeres son las trabas más comunes con las que las mujeres se topan.

- Por tanto, federaciones y clubes deben procurar becas de acompañamiento a todas las piragüistas de élite en la fase de maternidad y mientras se compita; se debe aumentar la visibilidad en los medios de comunicación del piragüismo femenino y generar espacios para atender a los hijos pequeños mientras se entrena y se compite.

- La hegemonía de estereotipos de belleza alejados del cuerpo de lapalista puede convertirse en motivo de abandono, implicando a menudo presiones por parte de padres y grupo de iguales que se traducen en falta de apoyo. Anivel educativo y mediático es necesario seguir avanzando en este sentido.

- Las federaciones deben hacer un esfuerzo especial en desarrollar acuerdos de colaboración entre clubes y otras instituciones (p.ej.: universidades), para favorecer la conciliación entre el piragüismo y los estudios, especialmente en categorías Junior y sub-23, pues el cambio de residencia y la imposibilidad de entrenar fuera de su localidad de origen es una de las mayores causas de abandono en estas categorías.

- Según los resultados obtenidos existe una imperiosa necesidad de exigir a las Federaciones y las Administraciones un mayor esfuerzo en la labor de promoción del piragüismo en general, y del piragüismo femenino en particular, trabajando en la mejora de la valoración social y la «visibilización» de las mujeres palistas.

\section{Referencias}

Alarcón, P. (2008). Mujer y Deporte: la actividad fiisica y el deporte de las mujeres andaluzas. Sevilla: Consejeriìa de Turismo, Comercio y Deporte.

Álvarez, P., Pérez, D., González, M., \& López, D. (2014). La formación universitaria de deportistas de alto nivel: análisis de una compleja relación entre estudios y deporte. RETOS. Nuevas tendencias en Educación Física, Deporte y Recreación, 26(2), 94-100.

Álvarez-Pérez, P., \& López-Aguilar, D. (2017). Modelo explicativo sobre trayectorias vitales y desarrollo vocacional en deportistas de alto nivel. RETOS. Nuevas tendencias en Educación Física, Deporte y Recreación, 0(34), 51-56.

Baena,A. \& Rebollo, S. (2008). Análisis del perfil sociodemográfico de la mujer como participante en raids de aventura. Retos. Nuevas tendencias en Educación Física, Deporte y Recreación, 14, 30-34.

Bianchi, S., \& Brinnitzer, E. (2000). Mujeres adolescentes y actividad física. Relación entre motivación para la práctica de la actividad física extraescolar y agentes socializadores, Digital, 5(26). Recuperado de http://www.efdeportes.com/Revista.

Castañeda, C., Zagalaz, M.L., Chacón, F., Cachón, J., \& Romero, S. (2014). Características de la práctica deportiva en función del género. Estudiantes de la Facultad de Ciencias de la Educación: Universidad deSevilla. RETOS. Nuevas tendencias en Educación Física, Deporte y Recreación, 25(1), 63-67.

Chillón, P.(2005). Efectos de un programa de intervención de educación física para la salud en adolescentes de $3^{\circ}$ ESO. (Tesis doctoral). Universidad de Granada, España.

Cruz, J. (1997). Psicología del deporte. Personalidad, evaluación y tratamiento psicológico. Madrid: Síntesis Psicología.

Edmuns, J., Ntoumanis, N., \& Duda, J. L. (2007). Adherence and wellbeing in overweight and obese patients referred to an exercise on pre- scription scheme: A self-determination theory perspective. Psychology of Sport and Exercise, 8, 722-740.

García Ferrando, M. (2005). Encuesta sobre hábitos deportivos de los españoles. Madrid: CIS

García Ferrando, M. (2006). Veinticinco años de análisis del comportamiento deportivo de la población española (1980-2005). Revista Internacional de Sociología (RIS), 64(44), 15-38.

Gómez, M., Ruiz, F., \& Pieron, M. (2010). Motivaciones en la práctica físi-co-deportiva del alumnado de secundaria. En Consejo Superior de De-portes (Ed.), Actividad físico-deportiva y salud. Análisis de los determinantes de la práctica en el alumnado de enseñanza secundaria. Madrid: CSD.

González, M. D., \& Torregrosa, M. (2009). Análisis de la retirada de la competición de élite: Antecedentes, transición y consecuencias. Revista Iberoamericana de Psicología del Ejercicio y el Deporte, 4(1), 93-104.

Isorna, M., Rial, A., \& Vaquero-Cristóbal, R. (2014). Motivaciones para la práctica deportiva en escolares federados y no federados. Retos. Nuevas tendencias en Educación Física, Deporte y Recreación, 25, 80-84.

Isorna, M., Ruiz, F., \& Rial, A. (2013). Variables predictoras del abandono de la práctica físico-deportiva en adolescentes. Cultura Ciencia Deporte, 8(23), 93-102. doi: 10.12800/ccd

Jiménez, M., Martínez P., Miró, E., \& Sánchez, I. (2008). Bienestar psicológico y hábitos saludables. International Journal of Clinical and Health Psychology, 8(1), 185-202.

Lavallee, D., \& Wylleman, P. (2000). Career transitions in sport. International perspectives (pp. 1-28). Morgantown, WV: Fitness Information Technology.

Latorre, P., García, F., \& López, J. (2017). Early sport dropout: High 
performance in early years in young athletes is not related with later success. Retos. Nuevas tendencias en Educación Física, Deporte y Recreación, O(33), 210-212.

Lee, A. M., Fredenburg, K., Belcher, D., \& Cleveland, N. (1999). Gender differences in children's conceptions of competence and motivation in physical education. Sport, Education \& Society, 4(2), 161-174.

Macarro, J, Romero, C., \& Torres, J. (2010). Motivos de abandono de la práctica de actividad físico-deportiva en los estudiantes de Bachillerato de la provincia de Granada. Revista de Educación, 353, 495-519.

Martínez, A., Chillón, P., Martín-Matillas, M., Pérez, I., Castillo, R., Zapatera, B., Vicente-Rodríguez, G., Casajús, J., Álvarez-Granda, L., Romero, C., Tercedor, P., \& Delgado-Fernández, M. (2012). Motives of Doprout and Non Practice Physical Activity and Sport in Spanish Adolescents: The Avena Study. Cuadernos de Psicología del Deporte, 12(1), 45-54.

Mollá, M. (2007). La influencia de las actividades extraescolares en los hábitos deportivos escolares. Revista internacional de medicina y ciencias de la actividad física y el deporte, 7(27), 241-252.

Moreno, J. A., Cervelló, E., \& Moreno, R. (2008). Importancia de la práctica físico-deportiva y del género en el autoconcepto físico de los 9 a los 23 años. International Journal of Clinical and Health Psychology, 8, 171-183.

Moreno, J. A., Martínez, C., \& Alonso, N. (2006). Actitudes hacia la práctica físico-deportiva según el sexo del practicante. Revista In ternacional de Ciencias del Deporte, 2(2), 20-43.

Moreno, J. A., Pardo, M., Jorge, P., \& Huéscar, E. (2016). Motivos de práctica físico-deportiva en mujeres: Diferencias entre practicantes y no practicantes. Revista de psicología del deporte, 25(1), 35-41.

Mota, J., \& Silva, G. (1999). Adolescent's physical activity: Association with socio-enconomic status and parental participation among a portuguese simple. Sport Educatio and Society, 4(2), 193-199.

Ogilvie, B.O., \& Howe, M. (1991). El trauma de la finalización de la vida deportiva. En J. M. Williams, (Ed.). Psicología aplicada al deporte (pp.523-548). Madrid: Biblioteca Nueva.

Orlick, T.D., \& Botterill, C.B. (1975). Every kid can win. Chicago, 1L: Nelson Hall.

Pallarès, S., Azócar, F., González, M. D., Korte, G., Mateos, M., Ramis, S., Palatnik, N., Stephan. Y., \& Torregrosa, M. (Marzo, 2008). El mismo deporte, dos realidades distintas. Comunicación al XI Congreso Nacional de Psicología de la Actividad Física y el Deporte. Sevilla.

Pallarès, S., Azócar, F., Korte, G., Ramis, Y., Fuentes, T. \& Torregrosa, M. (2009). Different trajectories, same sport: the case of waterpolo. Comunicación al XII ISSP World Congress of Sport Psychology. Marrakesh, Marroc.

Pallarès, S., Azócar, F., Torregrosa, M., Selva, C., \& Ramis, Y. (2011). Modelos de trayectoria deportiva en waterpolo y su implicación en la transición hacia una carrera profesional alternativa. Cultura, Ciencia y Deporte, 17(6), 93-103.

Pavón, A., \& Moreno, J. A. (2008). Actitud de los universitarios ante la práctica física-deportiva: diferencias por género. Revista de Psicología del Deporte, 17(1), 7-23.

Pavón, A., Moreno, J. A., Gutiérrez, M., \& Sicilia, A. (2004). Motivos de práctica físico-deportiva según la edad y el género en una muestra de universitarios. Apunts. Educación Física y Deportes, 76, 1321.

Piéron, M., Telama, R., Almond, L., \& Carreiro da Costa, F. (1999). Estilo de vida de jóvenes europeos: Un estudio comparativo. Revista de Educación Física, 76, 5-13.

Ponseti, F., García, A., Cantallops, J., \& Vidal, J. (2016). Diferencias de sexo respecto de la ansiedad asociada a la competición deportiva. Retos. Nuevas tendencias en Educación Física, Deporte y Recreación, 0(31), 193-196.

Puig, N. (2001). La situación de la mujer al iniciarse el siglo XXI. En M. Latiesa et al.(Eds.), Deporte y Cambio social en el umbral del siglo XXI (pp. 67-80). Madrid: Aeisad- Esteban.
Rodríguez,A. (2000). Adolescencia y deporte. Oviedo: Nobel.

Ruiz, F., Isorna, M., Ruiz-Risueño, J., \& Vaquero, R. (2014). Consumo e ingesta de alcohol en españoles mayores de 16 años y su relación con la actividad físico deportiva, la familia y el consumo de tabaco. Revista iberoamericana de psicología del ejercicio y el deporte, 9(2), 323-351.

Sallis, J., Prochaska, J., \& Taylor, W. (2000). A review of correlates of physical activity of children and adolescent. Medicine and Science in Sport and Exercise, 32(5), 963-975.

Saura, J. (1996). El entrenador en el deporte escolar. Lleida: Fundacioì Puiblica Institut d iEstudis Llerdencs.

Savin-Baden, M., \& Howell, C. (2013). Qualitative Research. The essential guide to theory and practice. New York: Routledge.

Scraton, S., \& Flintoff, A. (2002). Gender and sport: A reader. London: Routledge.

Selva, C., Pallarès, S., \& González, M.C. (2013). Una mirada a la conciliación a través de las mujeres deportistas. Revista de Psicología del Deporte, 22(1), 69-76.

Selva, C., Sahagún, M., \& Pallarès, S. (2011). Estudios sobre trayectoria profesional y acceso de la mujer a cargos directivos: un análisis bibliométrico. Revista de Psicología del Trabajo y de las Organizaciones, 27(3), 227-242.

Sousa, C., Cruz, J., Viladrich, C., \& Torregrosa, M. (2007). Efectos del programa de asesoramiento personalizado a entrenadores (PAPE) en el compromiso deportivo y el abandono de futbolistas jóvenes. Motricidad. European Journal of Human Movement, 19, 97-116.

Torre, E., Cárdenas, D., \& García, E. (2001). La motivación en la práctica físico deportiva. Lecturas: Educación Física y Deportes, Revista Digital, 39, Consulta: 03/06/16.

Torregrosa, M., Sánchez, X., \& Cruz, J. (2004). El papel del psicólogo del deporte en el asesoramiento académico-vocacional del deportista de élite. Revista de Psicología del Deporte, 13(2), 215-228.

Torregrosa, M., Sousa, C., Viladrich, C., Villamarín, F., \& Cruz, J.(2008). El clima motivacional y el estilo de comunicación del entrenador como predictores del compromiso en futbolistas jóvenes. Psicothema, 20(2), 254-259.

Tuero, C., \& Salguero, A. (2001a). El técnico deportivo y los deportes individuales I. Su rol en la iniciación deportiva. Natación, Saltos y Waterporlo. Revista de la Asociación Española de Técnicos de Natación, 23, 20-25.

Tuero, C., \& Salguero, A. (2001b). El técnico deportivo y los deportes individuales II. Su rol en las etapas de rendimiento deportivo. Natación, Saltos y Waterporlo. Revista de la Asociación Española de Técnicos de Natación, 24, 20-23.

Vilariño, M. F., \& Villar, C. L.(2012). La participación de las mujeres en el deporte: un análisis desde la perspectiva de género. En ICiclo de Conferencias Xénero, Actividade Física e Deporte, 2009-2010 (pp. 13-27).

Wurth, S., Lee, M. J., \& Alfermann, D. (2004). Parental involvement and athletes' career in youth sport. Psychology of Sport and Exercise, 5(1), 21-34.

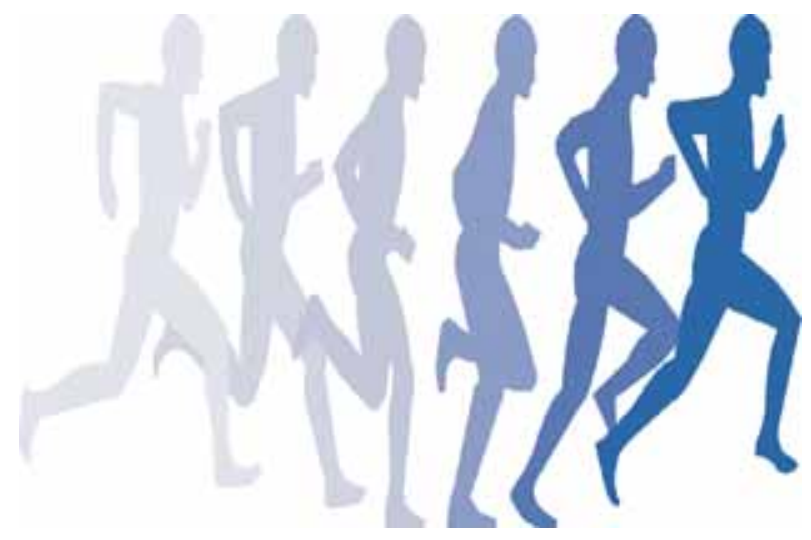

